\title{
Met-Activating Genetically Improved Chimeric Factor-1 Promotes Angio- genesis and Hypertrophy in Adult Myogenesis
}

\author{
Flavio Ronzoni ${ }^{1}$, Gabriele Ceccarelli ${ }^{2,3}$, Ilaria Perini ${ }^{4}$, Laura Benedetti ${ }^{2,3}$, Daniela Galli ${ }^{5}$, \\ Francesca Mulas ${ }^{3,6}$, Martina Balli ${ }^{2,3}$, Giovanni Magenes ${ }^{3,6}$, Riccardo Bellazzi, ${ }^{3,6}$, \\ Gabriella Cusella De Angelis ${ }^{2,3}$ and Maurilio Sampaolesi ${ }^{2,3,4, *}$
}

${ }^{1}$ Department of Pathology and Immunology, Faculty of Medicine, University of Geneva, Switzerland; ${ }^{2}$ Department of
Public Health, Experimental Medicine and Forensic, Faculty of Medicine, University of Pavia, Italy; ${ }^{3}$ Center of Health
Technologies (C.H.T), University of Pavia, Italy; ${ }^{4}$ Translational Cardiomyology Laboratory, Stem Cell Biology and
Embryology Unit, Department of Development and Regeneration, KU Leuven, Belgium; ${ }^{5}$ Department of Biomedical,
Biotechnological and Translational Sciences (S.Bi.Bi.T.), Anatomy and Histology Unit, University of Parma, Italy;
${ }^{6}$ Department of Industrial and Information, University of Pavia, Italy

Abstract: Background: Myogenic progenitor cells (activated satellite cells) are able to express both HGF and its receptor cMet. After muscle injury, HGF-Met stimulation promotes activation and primary division of satellite cells. MAGIC-F1 (Met-Activating Genetically Improved Chimeric Factor-1) is an engineered protein that contains two human Met-binding domains that promotes muscle hypertrophy. MAGIC-F1 protects myogenic precursors against apoptosis and increases their fusion ability enhancing muscle differentiation. Hemizygous and homozygous Magic-F1 transgenic mice displayed constitutive muscle hypertrophy.

\section{A R T I C L E H I S T O R Y}

Received: July 12,2016

Revised: November 03, 2016

Accepted: December 28, 2016

DOI:

$10.2174 / 13892010186661702011246$

02
Methods: Here we describe microarray analysis on Magic-F1 myogenic progenitor cells showing an altered gene signatures on muscular hypertrophy and angiogenesis compared to wild-type cells. In addition, we performed a functional analysis on Magic-F1 ${ }^{+/+}$transgenic mice versus controls using treadmill test.

Results: We demonstrated that Magic- $\mathrm{F}^{+/+}$mice display an increase in muscle mass and cross-sectional area leading to an improvement in running performance. Moreover, the presence of MAGIC-F1 affected positively the vascular network, increasing the vessel number in fast twitch fibers. Finally, the gene expression profile analysis of Magic-F $1^{+/+}$satellite cells evidenced transcriptomic changes in genes involved in the control of muscle growth, development and vascularisation.

Conclusion: We showed that MAGIC-F1-induced muscle hypertrophy affects positively vascular network, increasing vessel number in fast twitch fibers. This was due to unique features of mammalian skeletal muscle and its remarkable ability to adapt promptly to different physiological demands by modulating the gene expression profile in myogenic progenitors.

Keywords: Myogenic progenitor cells, recombinant proteins, muscle hypertrophy, angiogenesis, transgenic mice, microarray analysis.

\section{INTRODUCTION}

The growth of blood vessels from existing vasculature is known as angiogenesis and it is critical during the organ development and tissue repair. Recently, angiogenesis and compensatory muscle hypertrophy has been showed to be temporally combined, proposing that both processes could be regulated by common molecular mechanisms. VEGF

*Address correspondence to this author at the Translational Cardiomyology Laboratory, Stem Cell Biology and Embryology Unit, Department Development and Regeneration, KU Leuven, Belgium; Tel +32-(0) 16 330295; Fax+32-(0) 16 330294; E-mail: maurilio.sampaolesi@kuleuven.be
(Vascular Endothelial Growth Factor) and IGF (Insulin Growth Factor) play a major role in both angiogenesis and muscle hypertrophy $[1,2]$. However, the mechanism that regulates the recruitment of new blood vessels in hypertrophic muscles is not yet known in detail.

Angiogenesis is characterized by several biological features, including cell proliferation, migration, differentiation and cell-cell interaction $[3,4]$. The ability to control the angiogenic process could be directly stimulated by muscle hypertrophy [5]. VEGF is necessary for the proliferation and differentiation of endothelial precursor cells and conducts the association of endothelial cells into vascular structures (vas- 
culogenesis). Certain of these functions are essential for blood-vessel growth in normal tissue development and in tumor progression.

Muscular hypertrophy and atrophy are two contrasting mechanisms controlling the muscle mass, thinly regulated by the protein anabolism/catabolism [6, 7]. Physiologically, the activation of the hypertrophic pathway increases oxygen demand and promotes the formation of micro-capillaries to better sustain muscle contractile function. This suggests a crosstalk between myocytes and microvasculature in response to the hypertrophic induction, but also a spatiotemporal coordination between muscle growth/repair and angiogenesis [8].

Thus, the investigation about the pathways controlling muscle hypertrophy and angiogenesis could reveal novel possible therapeutic targets to counteract muscular diseases. Recent studies indicate that muscle degeneration observed in aged and dystrophic muscles can be contrasted by increasing muscle mass [7]. In this regard, MAGIC-F1 is a human recombinant protein derived from HGF that induces muscular hypertrophy [7, 9]. MAGIC-F1 is indeed involved in the cMet activation and in stimulating the anti-apoptotic cascade though Akt pathway activation. It has been demonstrated by previous studies that Magic-F1 transgenic mice show muscle hypertrophy and an increase in micro-capillaries during embryogenesis $[9,10]$.

Here we show the contribution of the recombinant protein MAGIC-F1 in promoting angiogenesis and hypertrophy in adult myogenesis. We perform morphometric, functional and vascular network analyses and compare the results to the gene signature obtained by Agilent microarray technology and validated through quantitative RT-PCR (qRT-PCR) analysis. Based on these features, MAGIC-F1 represents an important tool to promote muscle hypertrophy sustained by adequate vascularization and could be considered as an accessory transgene to improve cell-based therapies for muscle degenerations.

\section{MATERIALS AND METHOD}

\subsection{Animal Model: Magic-F1 Homozygous Mice}

Magic-F1 $1^{+/+}$mice were generated on a congenic FVB background, as previously described [9].

Mouse experiments were performed according to international ethical guidelines (EEC Council Directive 86/609; NIH Guide for the Care and Use of Laboratory Animals, 1985). The authorization for animal experimentation was obtained from the Italian Ministry of Health.

\subsection{Morphometric Analysis}

Six-month-old female mice were used and subjected to histological evaluation and morphometric analysis of tibialis anterior. Muscles obtained from transgenic and wt mice were embedded in OCT (Bio Optica) and cryosectioned for $10 \mu \mathrm{m}$ thickness. To define myocyte contour for morphometric analysis, slides were stained with Ematoxylin and Eosin (Bio Optica). Subsequently, samples were imaged using microscopy $(40 \mathrm{x})$ and analyzed to trace individual myocyte cross-sectional areas using ImageJ software. For statistical analysis, Mann-Whitney test was used.

\subsection{Treadmill Analysis}

Treadmill analyses were carried out twice a week for 4 weeks using a six-lane motorized treadmill (Exer 3/6 Treadmill; Columbus Instruments, Columbus, Ohio) supplied with shocker plates. The first trial was performed at low intensity and for short duration to accustom the mice to the exercise. The exercise load consisted of running at a speed of $5 \mathrm{~m} / \mathrm{min}$ for the first 5 minutes, after which the speed was increased $1 \mathrm{~m} / \mathrm{min}$ every 2 minutes. The test was stopped when the mouse remained on the shocker plate for more than $20 \mathrm{~s}$ without attempting to reengage the treadmill, and the time to exhaustion was determined. For statistical analysis, MannWhitney test was used.

\subsection{Vascular Network Analysis}

Capillary distribution was detected on muscle sections by indirect immunofluorescence with antibodies against the endothelial cell marker CD31 (PECAM - Santa Cruz Biotechnology, 1:200) and Smooth Muscle Actin (SMA Sigma-Aldrich). Arterioles and venules were localized by von Willebrand Factor (vWF-Abcam) and SMA immunostaining (1:250 and 1:250 respectively). Cell nuclei were counterstained in blue with DAPI. Two independent investigators counted capillary number in representative portions of each photograph. Comparisons were performed between Magic-F $1^{+/+}$and wild type mice with 6 mice per genotype evaluated. Data from the two independent investigators were averaged. For statistical analysis, Mann-Whitney test was used.

\subsection{Cell Cultures}

Satellite cells were maintained in DMEM supplemented with $2 \mathrm{mM}$ glutamine, $100 \mathrm{IU} / \mathrm{ml}$ penicillin, $100 \mu \mathrm{g} / \mathrm{ml}$ streptomycin and $10 \%$ FBS. All cultures were performed at $37^{\circ} \mathrm{C}$ in a humidified incubator with 5\% CO2 and 95\% air. Satellite cells were prepared following the protocol of Dellavalle et al. [11]. Cell differentiation was carried out for 3 days. Cells were grown on $10 \mathrm{~cm}$ Petri dishes until sub-confluence.

\subsection{RNA Extraction and Microarray Analysis}

All microarrays were performed on Agilent platform (Agilent Quick Amp 1color) and analyzed by the VIB Microarray Facility (www.microarray.be). Biological triplicates of Magic-F1 ${ }^{+/+}$and control (MLC3F) satellite cells were used for microarray comparison. Total RNA was isolated using a standard extraction protocol with Quiagen RNeasy Plus Mini Kit (50) following manufacturer instructions. RNA was quantified using a NanoDrop (Thermo Scientific) spectrophotometer. Differences in gene expression were evaluated on a single-color Agilent chip. Data analysis is based on the expression values as obtained from the Agilent Feature Extraction (FE Software version 10.5.1.1)

\subsection{Microarray Validation}

Validation of altered gene expression was performed using SYBR Green qRT-PCR using the same total RNA 
used for the microarray analyses. qRT-PCR analysis has been performed on genes involved in hypertrophy and atrophy pathway using the following primers: MyoD (Myogenic differentiation 1), MSTN (Myostatin), FST (Follistatin), FoxO (Forkhead box O), MyoG (Myogenin), Notch-1, PAX7 (Paired box 7), PAX3 (Paired box 3), IGF-1, VEGF- $\alpha$ and VEGF- $\beta$. Gene expression was analysed in triplicate and normalised to the CT mean of GAPDH (Glyceraldehyde-3phosphate dehydrogenase) gene expression. Primers for the genes are listed in Table $\mathbf{1}$

\subsection{Statistical Analysis}

Data are shown as mean \pm S.E.M. Comparison between 2 groups were performed in triplicates and statistically analyzed using a paired 2-tailed Student $t$ test. P-values $<0.05$ were considered significant.

\section{RESULTS}

\subsection{Morphometric and Functional Analyses of Magic- $\mathrm{F}^{+/+}$Mice}

Morphometric analysis revealed an increase of the cross sectional area in muscle fibres of Magic-F $1^{+/+}$mice. In 5 months old mice, tibialis sections showed a statistically significant increase of the area and perimeter of muscle fibres compared to wt age-matched animals ( 9 mice per group, 300-460 fibres analyzed for each sample) (Fig. 1A,B); meanwhile no differences were observed in slow-twitch muscles such as soleus (Fig. 1C,D).
Consistent with the idea that muscle hypertrophy would affect the performance of Magic-F $1^{+/+}$mice, transgenic mice were subjected to an in vivo motility assay, the treadmill exhaustion test. On average, homozygous mice covered a longer distance compared to wt age-matched animals (Fig. 1E), moreover the time for exhaustion was increased (Fig. 1F), proving that Magic-F1 expression strengthened the physical performance, predisposing the animal to more intense efforts.

\subsection{Vascular Network in Magic-F1 ${ }^{+/+}$Skeletal Muscle}

During muscular hypertrophy, the increase of muscle mass must be counterbalanced by an increase of the capillary network in order to provide further oxygen and growth supply.

Taken these considerations, we evaluated changes in the capillary supply network by counting the number of PECAM positive cells in different sections of tibialis anterior (Fig. 2A,B) and soleus (Fig. 2E,F) coming from 5-6 months old Magic-F ${ }^{+/+}$and wt mice. The average number of capillaries per fibre and per $\mathrm{mm}^{2}$ surface area in wt and Magic-F1 $1^{+/+}$ muscles is reported in Table $\mathbf{2}$, showing a higher capillary content in transgenic muscles.

Immunostaining revealed a higher number of vWF (von Willebrand factor) and SMA (Smooth Muscle Actin) double positive cells in Magic-F1 ${ }^{+/+}$tibialis anterior muscles compared to controls (Fig. 2C,D). Further statistical analysis confirmed a higher capillary distribution around muscle fibres in transgenic mice compared to controls. In addition,

Table 1. Primer sequences used in qRT-PCR.

\begin{tabular}{|c|l|l|}
\hline Primer & \multicolumn{1}{|c|}{ Forward } & Reverse \\
\hline \hline GAPDH* & GGTCGGAGTGAACGGATTTG & CAACGATGTCCACTTTGCCA \\
MyoD & TGCACTTCCACCAACCCCAACCAGC & CCTGGACTCGCGCACCGCCTCACT \\
Myostatin & AGTGACGGCTCTTTGGAAGATG & AGTCAGACTCGGTAGGCATGGT \\
Follistatin & CTGTACAAGACCGAACTGAGC & TCCACAGTCCACGTTCTCACA \\
FoxO & TGTTTGCTGATGGCAACTTC & GATCAGCTCGGGCACTTTAG \\
MyoG & TGTTTGCTGATGGCAACTTC & GATCAGCTCGGGCACTTTAG \\
Notch-1 & TGTTTGCTGATGGCAACTTC & GATCAGCTCGGGCACTTTAG \\
Pax7 & GGGATGCCTTTGTGGAACTA & CTCACTTGTGGCCCAGGTAT \\
Pax3 & AGGAGGCGGATCTAGAAAGGAAG & TGTGGAATAGACGTGGGCTGGTA \\
IGF-1 & CTGTGCCCCACTGAAGCCTA & GGACTTCTGAGTCTTGGGCATG \\
VEGF- $\boldsymbol{\alpha}$ & TGTTTGCTGATGGCAACTTC & GATCAGCTCGGGCACTTTAG \\
*Housekeeping gene & TGTTTGCTGATGGCAACTTC & GATCAGCTCGGGCACTTTAG \\
\hline
\end{tabular}

Table 2. Vessel network distribution.

\begin{tabular}{|c|c|c|c|}
\hline & N. Capillaries per Fibre & N. Capillaries per $\mathbf{m m}^{2}$ & N. Small vessels per mm $^{2}$ \\
\hline \hline Magic-F1 $^{+++}$ & $5(+/-0,18)$ & $20091(+/-17)$ & $45(+/-5)$ \\
\hline wt & $4(+/-0,3)$ & $2008(+/-21)$ & $26(+/-10)$ \\
\hline
\end{tabular}


A

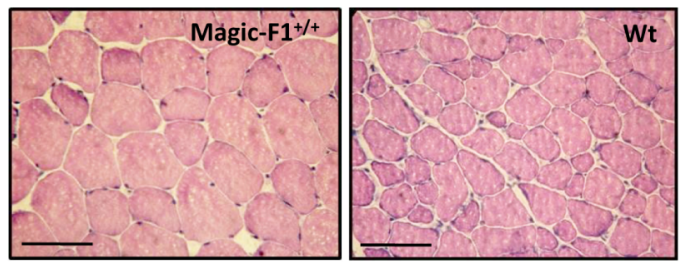

B

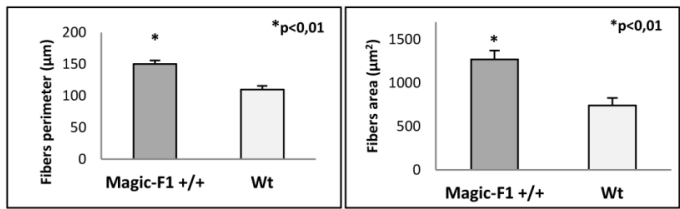

C

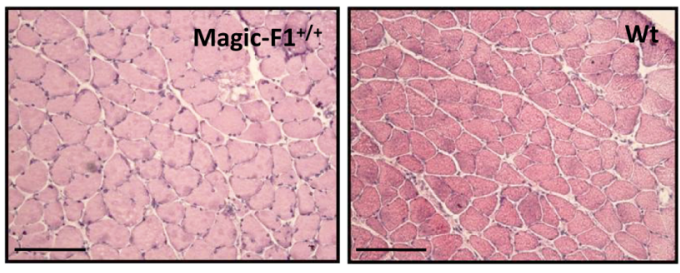

D

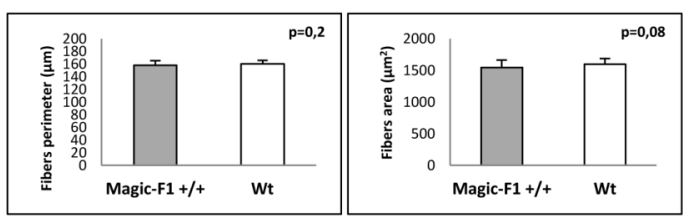

E
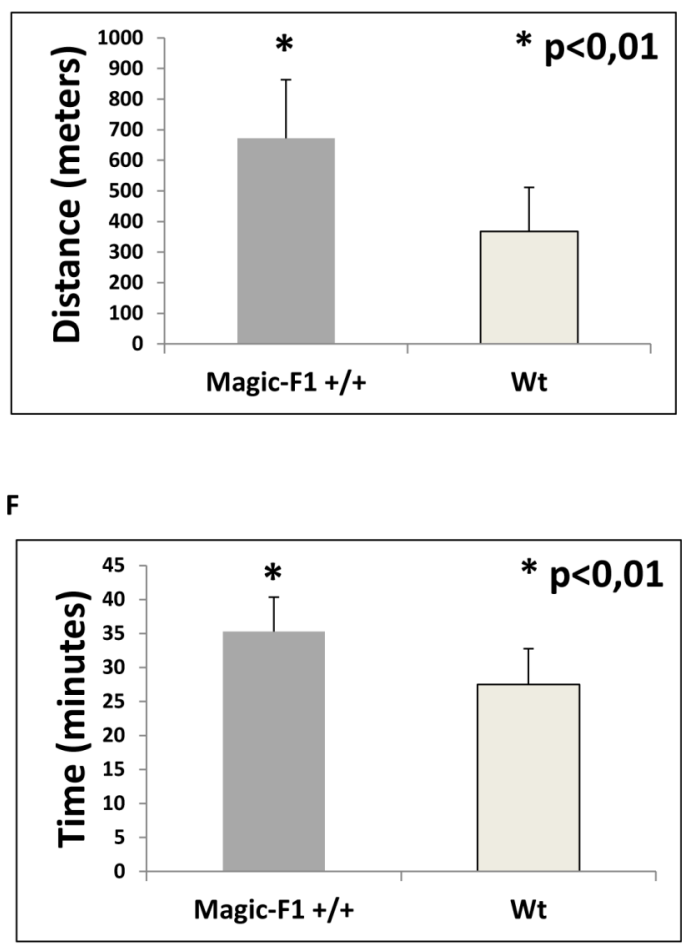

Fig. (1). Morphometrical and treadmill analysis. (A) Fibres section of tibialis anterior in Magic-F $1^{+/+}$(left panel) and wt mice (right panel). (B) Morphometrical analysis performed on tibialis anterior shows an increase in perimeter and area of muscle fibres in Magic-F1 ${ }^{+/+}$ compared to control mice. (C) Fibres section of soleus in Magic-F1 ${ }^{+/+}$(left panel) and wt mice (right panel). (D) Morphometrical analysis performed on soleus shows similar size of Magic-F1 ${ }^{+/+}$and wt muscle fibres. (E,F): Exhaustion treadmill tests carried on Magic-F1 ${ }^{+/+}$transgenic mice (gray label) and wt mice (white label). The results are presented as the mean \pm standard error of the mean (S.E.M.).

tibialis anterior muscles of transgenic mice contained an average of 5 capillaries per muscle fibre (Fig. 2A) and an increased total number of capillaries (Fig. 2B) and small vessels (Fig. 2C, D) per $\mathrm{mm}^{2}$. Similar analyses were performed on soleus muscles, but no statistical differences were observed in capillary total number and distribution between Magic-F $1^{+/+}$and wt mice (Fig. 2E, 2F, 2G, 2H). Nevertheless, due to the increased capillary network, we speculate that a better blood and oxygen supply is required in the hypertrophic muscles of transgenic mice.

\subsection{Microarray-based Gene Expression Profile}

In order to investigate the molecular basis of the increase of muscle mass and vascularization in Magic- $F 1^{+/+}$mice, we evaluated the gene signature of muscle progenitor cells by microarray analysis (Agilent Quick Amp 1color) (Suppl. Fig. 1A). Gene expression profiling evidenced important transcriptomic changes, with several up- and down-regulated genes in transgenic muscle progenitor cells compared to MLC3F controls (GEO\# GSE83771). The analysis of differentially expressed genes by Gene Ontology categories revealed a significant $(p<0.05)$ enrichment in functional categories related to muscle cell fate commitment, regulation of muscle contraction, muscle adaptation, and muscle cell migration (Suppl. Fig. 1C). Interestingly, Magic-F1 seems to modulate the expression of genes involved in the control of muscle growth and development, such as MyoD, MSTN, FST, FoxO, MyoG, Notch1 and PAX7 (Suppl Fig. 2A). Consistent with the increased vascularization of transgenic muscles, the expression of a number of genes involved in angiogenesis was altered, including IGF and VEGF $\beta$ growth factors (Suppl Fig. 2B). We further analyzed the transcriptomic effects of Magic-F1 on muscle progenitor cells (mpcs) of adult transgenic mice by KEGG pathways and networks. Among the biological functions that showed highly significant $(\mathrm{P}<0.00001)$ changes in Magic-F1 ${ }^{+/+}, \mathrm{VEGF}$, mTOR (mechanistic target of rapamycin), MAPK (mitogenactivated protein kinase), cardiac muscle contraction and hypertrophic cardiomyopathy signalling pathways were enriched with 74, 46, 254, 69 and 79 differentially expressed genes, respectively (Suppl. Fig. 1D).

\subsection{Microarray Analysis Validation}

Microarray results were validated by qRT-PCR (Biorad MiniOpticon System) and 11 genes were chosen to represent both upregulated and downregulated genes with a defined range of different expression levels. The experimental conditions applied in the microarray analysis were maintained during validation. Importantly, results obtained by qRT-PCR were in agreement with microarray analysis. Complete lists 
A
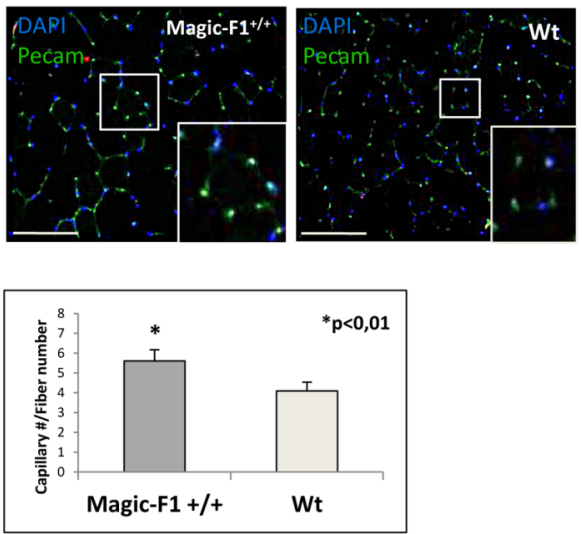

C
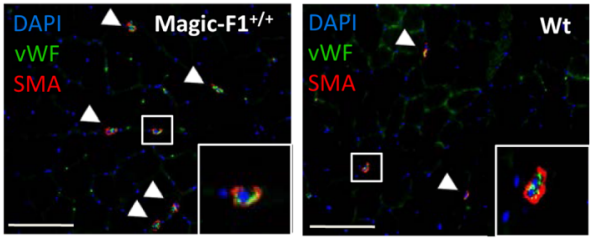

D

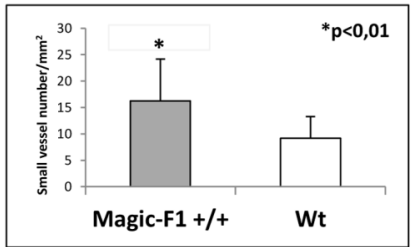

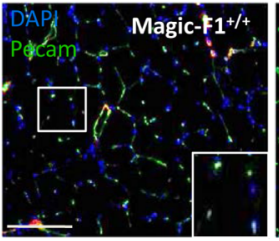

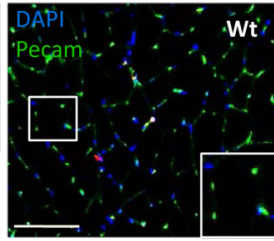

$\mathbf{F}$

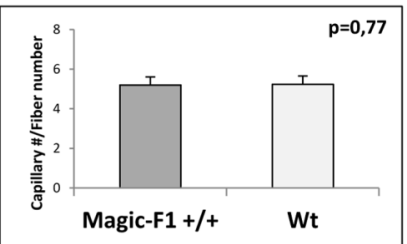

G

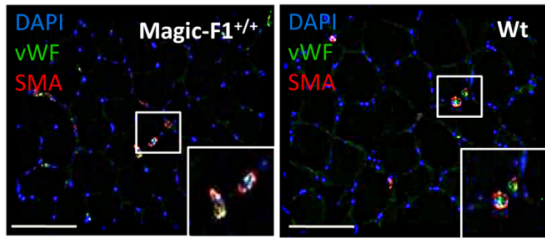

H

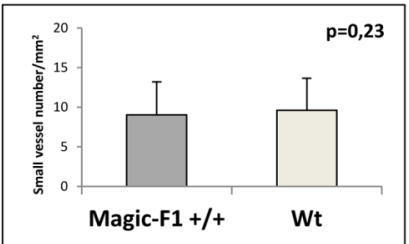

Fig. (2). Vascular network analysis. (A) PECAM (CD31+) immunofluorescence signal (green) shows a higher capillary density in Magic$\mathrm{F} 1^{+/+}$tibialis anterior (left panel) compared to control (right panel); nuclei were stained in blue with DAPI. (B) Capillary number per fibre cross-sectional area was much greater in Magic-F1 ${ }^{+/+}$than in wt muscle. (C) vWF (green) and SMA (red) staining were used to quantify the number of small vessels in Magic-F1 $1^{+/+}$(left panel) and wt (right panel) tibialis anterior. (D) Double positive staining vWF/SMA shows that the number of small vessels was higher in Magic-F $1^{+/+}$mice compared to control. E) PECAM (CD31+) immunofluorescence signal (green) shows a similar capillary density in Magic-F1 $1^{+/+}$soleus (left panel) compared to control (right panel); nuclei were stained in blue with DAPI. (F) Capillary number per fibre cross-sectional area was not statistically significant between Magic-F $1^{+/+}$and wt muscle. (G) vWF (green) and SMA (red) staining were used to quantify the number of small vessels in Magic-F1 ${ }^{+/+}$(left panel) and wt (right panel) soleus. (H) Double positive staining $\mathrm{VWF} / \mathrm{SMA}$ shows that there is no difference between the number of small vessels in Magic-F $1^{+/+}$and control muscles.

Table 3. Validation of the microarray data in proliferating satellite cells by qRT-PCR.

\begin{tabular}{|c|c|c|c|}
\hline \multirow[b]{2}{*}{ Gene Symbol } & \multicolumn{3}{|c|}{ qRT-PCR Expression Analysis During Proliferation Process } \\
\hline & Magic-F1 $^{+/+}$ & wt & P-value \\
\hline MyoD & $-1,07$ & $-2,39$ & $\mathbf{P}<0,01$ \\
\hline MSTN & 6,02 & 3,94 & $\mathrm{P}<0,01$ \\
\hline FST & $-1,64$ & $-2,37$ & $\mathrm{P}<0,05$ \\
\hline MyoG & 4,8 & $-0,9$ & $\mathrm{P}<0,01$ \\
\hline Notch1 & $-0,47$ & $-0,18$ & $\mathrm{P}<0,05$ \\
\hline PAX7 & 1,55 & 0,02 & $\mathrm{P}<0,01$ \\
\hline PAX3 & 8,45 & 8,68 & $\mathrm{P}=0,08$ \\
\hline
\end{tabular}


of differentially expressed genes resulting from microarray analysis of Magic-F $1^{+/+}$and control MLC3F satellite cells with a $\mathrm{P}<0.05$ were considered statistically significant as shown in (Table 3 ).

Notably, several genes presented in the table are critical for the muscle growth and function. These included MyoD, MSTN, FST, FoxO, MyoG, Notch1, Pax3-7 and growth factors implicated in skeletal muscle development and angiogenesis, including IGF, VEGF- $\alpha$ and VEGF- $\beta$.

During proliferation we observed in Magic- $1^{+/+}$satellite cells a statistically significant decrease in the expression of some genes involved in muscle differentiation like MyoD, MyoG and Pax7, three factors normally expressed in activated satellite cells. Other down-regulated genes were MSTN and FST, both involved in muscle growth (Fig. 3A). These results allowed us to hypothesize that mpcs derived from Magic-F $1^{+/+}$mice have a higher proliferative capacity, at least partly responsible for muscle hypertrophy. IGF stimulates cell proliferation and inhibits apoptosis while VEGF $\beta$ promotes endothelial cell proliferation, thereby stimulating angiogenesis. These two growth factors appeared to be upregulated in Magic-F1 $1^{+/+}$satellite cells (Fig. 3B). Taken together, the results clearly indicated that Magic-F $1^{+/+}$ mpcs have a high proliferation ability, a reduced sensitivity to apoptosis and a proangiogenic signal activation compared to the MLC3F controls. Afterwards we performed a comparative analysis during differentiation, evaluating the ex- pression of selected genes in Magic-F1 ${ }^{+/+}$and control mpcs (Table 4). Notch1, PAX3 and PAX7 resulted upregulated in Magic-F ${ }^{+/+}$satellite cells compared to control, while MyoD, a late marker of differentiation, MSTN, FST and MyoG were strongly downregulated (Fig. 3C). The results indicate that 6 out of the 11 analyzed genes involved in muscle differentiation were upregulated in Magic-F $1^{+/+}$cells compared to controls, explaining at least in part the Magic-F1 ${ }^{+++}$muscle hypertrophic phenotype. MSTN and FST expressions were downregulated but not IGF-1 (Fig. 3D), suggesting that a possible crosstalk between MAGIC-F1 and other pathways could also exist. This is in agreement with previous studies showing that MSTN ablation promoted myoblast differentiation and muscle hypertrophy [12].

\subsection{Knowledge-based Gene Association Networks for Exploring Muscle Hypertrophy Gene Signature}

Bioinformatic analysis of microarray data led us to the identification of gene regulatory networks and biochemical pathways involved in muscle hypertrophy and angiogenesis. These tests were conducted with Python scripts that integrate statistical analysis with functions provided by the Data Mining and Bioinformatics software Orange and with other gene-related information available from biological repositories. A pipeline for building knowledge-based gene association networks [13] has been applied to the transcriptional analysis of mpcs. First, a selection procedure allowed us to
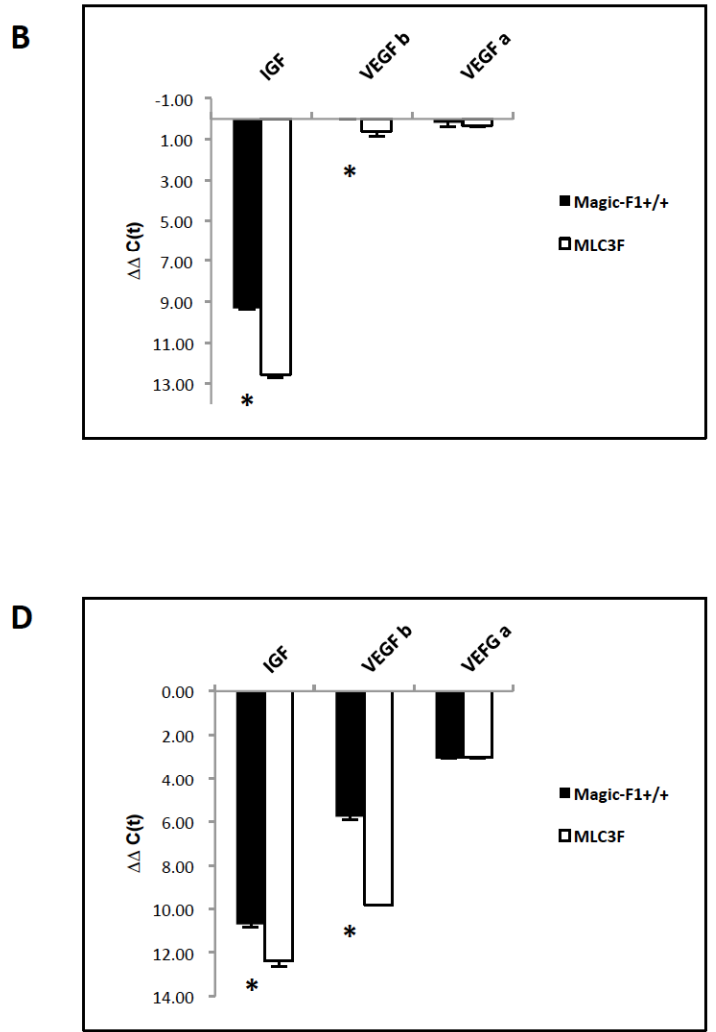

Fig. (3). Gene expression signature. Histograms illustrating qRT-PCR analysis of up-regulated and down-regulated muscle specific genes (A) and growth factors (B) in Magic-F1 $1^{+/+}$and control (MLC3F) undifferentiated satellite cells. qRT-PCR analysis of muscle specific genes (C) and growth factors (D) on differentiated satellite cells of Magic-F1 $1^{+/+}$and control (MLC3F) mice. Values are normalized to the level of GAPDH, $n=5$. 
Table 4. qRT-PCR analysis in differentiated satellite cells.

\begin{tabular}{|c|c|c|c|}
\hline \multirow[b]{2}{*}{ Gene Symbol } & \multicolumn{3}{|c|}{ qRT-PCR Expression Analysis During Differentiation Process } \\
\hline & Magic-F1 $^{+/+}$ & wt & P-value \\
\hline MyoD & $-0,87$ & 0,93 & $\mathrm{P}<0,01$ \\
\hline MSTN & 12,51 & 5,88 & $\mathrm{P}<0,01$ \\
\hline FoxO & 5,94 & 7,93 & $\mathrm{P}<0,05$ \\
\hline MyoG & 13,62 & 11,46 & $\mathrm{P}<0,05$ \\
\hline Notch1 & 2,88 & 4,92 & $\mathrm{P}<0,05$ \\
\hline PAX7 & 5,55 & 10,63 & $\mathrm{P}<0,01$ \\
\hline VEGFa & 3,08 & 3,04 & $\mathrm{P}=0,06$ \\
\hline
\end{tabular}

identify several genes differentially expressed $(\mathrm{p}<0.05)$ in Magic-F ${ }^{+/+}$mice versus controls including transcription and growth factors (Table 3). Starting from these sets of musclespecific genes and growth factors, we constructed functional annotation gene networks where links indicated a high similarity of their annotations, as recorded on MeSH and Gene Ontology databases. For each selected gene, its 5 most similar genes in the mouse genome were added to the initial list of molecules used for constructing the network. This step allowed including in the analysis other genes that are likely to be involved in the same processes. The final network was obtained by connecting pairs of genes in the expanded list for which the annotation similarity exceeded a cut-off of 0.7 . The visualization of these networks identified wellcharacterized genes involved in skeletal muscle biology, such as MSTN, FST, MyoG, MyoD and Myf5 (Suppl. Fig. 1B). These clusters also highlighted genes having a potential novel role in muscle hypertrophy. In particular, the network underlined the significant differential expression of Vestigial-like 2 (Vgll2), not initially selected as a focus gene but added later, due to its association with MyoD. Vgll2 is a muscle-specific cofactor able to promote skeletal muscle differentiation and the activation of muscle-specific promoters. Vgll2 is required for muscle gene expression during muscle differentiation [14]. The role of this candidate gene and others is currently being investigated.

In conclusion, MAGIC-F1 signalling has not formerly been investigated in transgenic mice; however, alterations in myoblast differentiation and muscle hypertrophy-driven tissue reshaping would undoubtedly be related to the prevalence of these pathways.

\section{DISCUSSION}

This study was conducted to better understand the myogenic and angiogenic contributions to the muscle hypertro- phy observed in Magic-F1 $1^{+/+}$transgenic mice. We focused our attention on identifying relevant patterns of gene expression involved in muscle hypertrophy and might therefore account for the muscle angiogenesis. In the developing muscle tissue, the expression of Magic-F1 was shown to overlap with PAX3 [10]. Here we showed that Magic-F1 could induce muscle hypertrophy and angiogenesis by both downregulating myostatin, and directly activating IGF and VEGF pathways (Fig. 3). These characteristics could be of interest for many potential therapeutic applications. Indeed, many cell types express the cMet receptor, including epithelial cells of several organs $[15,16]$. Additionally, cMet is commonly upregulated in several human cancer cells [17], suggesting its critical role in promoting tumor progression [18]. Thus, MAGIC-F1 could be tested in cMet positive tumor cells as cMet partial agonist incapable of inducing cell proliferation.

With the growing number and the increasing reliability of biological knowledge bases, bioinformatics and functional genomics have recently shifted from the application of dataoriented methods to the study of procedures where prior knowledge is included in the data analysis process. In order to analyze high-throughput data (DNA microarray), the most widely used approach focuses on gene sets such as Gene Ontology and KEGG, which are based on the information obtained from biological repositories [19]. The gene annotation terms extracted from the various knowledge repositories can be exploited to visualize gene similarities by means of graphs called gene association networks, where each gene is a node in the graph and a connecting link is created between every pair of genes that are similar in terms of annotations.

In this work, we applied a procedure that, given a set of genes of interest ('focus genes'), suggests the creation of gene association networks where the focus genes are connected to their annotation-similar genes according to the 
Gene Ontology and the Medical Subject Headings from NCBI's PubMed (Suppl. Fig. 1B). The network has identified several genes affected in Magic-F ${ }^{+/+}$hypertrophic mice, such as VEGF, mTOR, MAPK and some others related to biological processes and pathways involved in cardiac muscle contraction and in hypertrophic cardiomyopathy (Suppl Fig. 1C,D), further supporting the phenotype of Magic-F $1^{+/+}$ hypertrophic mice.

The procedure has been applied to a transcriptional analysis where, starting from a list of muscle specific genes and muscle growth regulators, we obtained networks that emphasize the results of global transcriptome analysis for the focus genes and their annotation-neighbours. In addition, our strategy allowed us to identify novel candidate genes potentially involved in muscle hypertrophy and angiogenesis such as Vgll2 and Church1 that require further investigation (Suppl. Fig. 1B).

Although it has been previously described that HGF inhibits skeletal muscle differentiation $[20,21]$, other authors showed that HGF administration induces hypertrophy in cardiomyocytes $[22,23]$. Thus, it is not surprising the similar effect of Magic-F1 in transgenic skeletal muscles. However, additional experiments are required to clarify how MAGICF1 could alter the cMet signalling and mimic the observed HGF effects in cardiomyocytes.

New blood vessels and oxygen supply are crucial to improve the efficacy of stem cell-based therapeutic protocols. Its ability to promote angiogenesis makes MAGIC-F1 a potential candidate for muscle degenerative disorders where the microcirculation is compromised. Due to its small cDNA size (approximately $1.4 \mathrm{~kb}$ ), Magic-F1 may be used alone or as an additional adjuvant factor in gene therapy protocols [24]. Previous studies showed the ability of mesoangioblasts, vessel associated stem cells, to contribute directly to muscle regeneration in animal models of muscular dystrophies [25, 26]. In addition, a phase I/II clinical trial proved safety of intra-arterial mesoangioblast transplantation but it was not efficacious [27]. Thus implementation of the cell therapy protocol is desirable. In this view MAGIC-F1 can be considered in cell therapy protocols to improve stem cell migration and myogenic contribution aiming at advancing towards efficacy.

\section{CONFLICT OF INTEREST}

The author(s) confirm that this article content has no conflict of interest.

\section{ACKNOWLEDGEMENTS}

F.R. G.C. and M.S. designed research and drafted the ms; I.P., D.G., F.M., and M.B. performed experiments; L.B., R.B., G.M., F.R., G.C., G.C.D. and M.S analyzed data, contributed to critical experiments/insights; G.C.D. and M.S. provided funding. This work has been funded by "Opening The Future" Campaign (EJJ-OPTFUT-02010), CARE-MI FP7, AFM, CARIPLO\#2015_0634, FWO\#G060612N, FWO\#G0A8813N, FWO\#G0887̄15N GOA, IUAP, NATO RAWINTS\#G984961 and Project financiering Stem Cells
(PFO3 10/019) grants. We thank Luigi Vercesi, Christina Vochten and Vicky Raets for professional administrative assistance. We would also like to thank Rondoufonds voor Duchenne Onderzoek for a kind donation.

\section{SUPPLEMENTARY MATERIAL}

Supplementary material is available on the publisher's web site along with the published article.

\section{REFERENCES}

[1] Pillai V.B.; Sundaresan N.R.; Gupta M.P. Regulation of Akt signaling by sirtuins: its implication in cardiac hypertrophy and aging. Circ res, 2014, 114(2), 368-378.

[2] Rabinovsky E.D. The multifunctional role of IGF-1 in peripheral nerve regeneration. Neurol res 2004, 26(2), 204-210.

[3] Sandri M.; Barberi L.; Bijlsma A.Y.; Blaauw B.; Dyar K.A.; Milan G.; Mammucari C.; Meskers C.G.; Pallafacchina G.; Paoli A.; Pion D.; Roceri M.; Romanello V.; Serrano A.L.; Toniolo L.; Larsson L.; Maier A.B.; Muñoz-Cánoves P.; Musarò A.; Pende M.; Reggiani C.; Rizzuto R.; Schiaffino S. Signalling pathways regulating muscle mass in ageing skeletal muscle: the role of the IGF1Akt-mTOR-FoxO pathway. Biogerontology 2013, 14(3), 303-323.

[4] Jones N.; Iljin K.; Dumont D.J.; Alitalo K. Tie receptors: new modulators of angiogenic and lymphangiogenic responses. Nat rev Mol cell biol 2001, 2(4), 257-267.

[5] Palstra A.P.; Rovira M.; Rizo-Roca D.; Torrella J.R.; Spaink H.P.; Planas J.V. Swimming-induced exercise promotes hypertrophy and vascularization of fast skeletal muscle fibres and activation of myogenic and angiogenic transcriptional programs in adult zebrafish. BMC genomics 2014, 15,1136.

[6] Sandri M. Signaling in muscle atrophy and hypertrophy. Physiology 2008, 23,160-170

[7] Cassano M.; Quattrocelli M.; Crippa S.; Perini I.; Ronzoni F.; Sampaolesi M. Cellular mechanisms and local progenitor activation to regulate skeletal muscle mass. J muscle res cell motil $\mathbf{2 0 0 9}$, 30(7-8), 243-253.

[8] Bentzinger C.F.; Wang Y.X.; Dumont N.A.; Rudnicki M.A. Cellular dynamics in the muscle satellite cell niche. EMBO reports $\mathbf{2 0 1 3}$ 14(12),1062-1072

[9] Cassano M.; Biressi S.; Finan A.; Benedetti L.; Omes C.; Boratto R.; Martin F.; Allegretti M.; Broccoli V.; Cusella De Angelis G.; Comoglio P.M.; Basilico C.; Torrente Y.; Michieli P.; Cossu G.; Sampaolesi M. Magic-factor 1, a partial agonist of Met, induces muscle hypertrophy by protecting myogenic progenitors from apoptosis. PloS One 2008, 3(9), e3223.

[10] Ronzoni F.; Bongio M.; Conte S.; Vercesi L.; Cassano M.; Tribioli C.; Galli D.; Bellazzi R.; Magenes G.; Cusella De Angelis M.G.; Sampaolesi M. Localization of Magic-F1 transgene, involved in muscular hypertrophy, during early myogenesis. J Biomed Biotechnol 2011, 2011,492075.

[11] Dellavalle A.; Sampaolesi M.; Tonlorenzi R.; Tagliafico E.; Sacchetti B.; Perani L.; Innocenzi A.; Galvez B.G.; Messina G.; Morosetti R.; Li S.; Belicchi M.; Peretti G.; Chamberlain J.S.; Wright W.E.; Torrente Y.; Ferrari S.; Bianco P.; Cossu G. Pericytes of human skeletal muscle are myogenic precursors distinct from satellite cells. Nat Cell Biol 2007, 9(3), 255-267.

[12] McPherron A.C.; Lawler A.M.; Lee S.J. Regulation of skeletal muscle mass in mice by a new TGF-beta superfamily member. $\mathrm{Na}$ ture 1997, 387(6628), 83-90.

[13] Zuccotti M.; Merico V.; Bellone M.; Mulas F.; Sacchi L.; Rebuzzini P.; Prigione A.; Redi C.A.; Bellazzi R.; Adjaye J.; Garagna S Gatekeeper of pluripotency: a common Oct4 transcriptional network operates in mouse eggs and embryonic stem cells. BMC genomics 2011, 12,1-13.

[14] Chen H.H.; Maeda T.; Mullett S.J.; Stewart A.F. Transcription cofactor Vgl-2 is required for skeletal muscle differentiation. Genesis 2004, 39(4), 273-279.

[15] Trusolino L.; Comoglio P.M. Scatter-factor and semaphorin receptors: cell signalling for invasive growth. Nat Rev Cancer 2002, 2(4), 289-300 
[16] Birchmeier C.; Birchmeier W.; Gherardi E.; Vande Woude G.F. Met, metastasis, motility and more. Nat Rev Mol Cell Biol 2003, 4(12), 915-925.

[17] Corso S.; Comoglio P.M.; Giordano S. Cancer therapy: can the challenge be MET? Trends Mol Med 2005, 11(6),284-292.

[18] Takahara T.; Xue F.; Mazzone M.; Yata Y.; Nonome K.; Kanayama M.; Kawai K.; Pisacane A.M.; Takahara S.; Li X.K.; Comoglio P.M.; Sugiyama T.; Michieli P. Metron factor-1 prevents liver injury without promoting tumor growth and metastasis. Hepatology 2008, 47(6), 2010-2025.

[19] Subramanian A.; Tamayo P.; Mootha V.K.; Mukherjee S.; Ebert B.L.; Gillette M.A.; Paulovich A.; Pomeroy S.L.; Golub T.R.; Lander E.S.; Mesirov J.P. Gene set enrichment analysis: a knowledge-based approach for interpreting genome-wide expression profiles. Proc Natl Acad Sci U S A 2005, 102(43), 15545-15550.

[20] Gal-Levi R.; Leshem Y.; Aoki S.; Nakamura T.; Halevy O. Hepatocyte growth factor plays a dual role in regulating skeletal muscle satellite cell proliferation and differentiation. Biochim et Biophys acta 1998, 1402(1), 39-51.

[21] Leshem Y.; Spicer D.B., Gal-Levi R.; Halevy O. Hepatocyte growth factor (HGF) inhibits skeletal muscle cell differentiation: a role for the bHLH protein twist and the cdk inhibitor p27. J Cell Physiol 2000, 184(1), 101-109.

[22] Li Y.; Takemura G.; Kosai K.; Yuge K.; Nagano S.; Esaki M.; Goto K.; Takahashi T.; Hayakawa K.; Koda M.; Kawase Y.; Maruyama R.; Okada H.; Minatoguchi S.; Mizuguchi H.; Fujiwara T.; Fujiwara H.; Postinfarction treatment with an adenoviral vector expressing hepatocyte growth factor relieves chronic left ventricular remodeling and dysfunction in mice. Circulation 2003, 107(19), 2499-2506.
Fiaccavento R.; Carotenuto F.; Minieri M.; Fantini C.; Forte G.; Carbone A.; Carosella L.; Bei R.; Masuelli L.; Palumbo C.; Modesti A.; Prat M.; Di Nardo P. Stem cell activation sustains hereditary hypertrophy in hamster cardiomyopathy. $J$ Pathol 2005 , 205(3), 397-407.

[24] Minetti G.C.; Colussi C.; Adami R.; Serra C.; Mozzetta C.; Parente V.; Fortuni S.; Straino S.; Sampaolesi M.; Di Padova M.; Illi B.; Gallinari P.; Steinkühler C.; Capogrossi M.C.; Sartorelli V.; Bottinelli R.; Gaetano C.; Puri P.L. Functional and morphological recovery of dystrophic muscles in mice treated with deacetylase inhibitors. Nat Med 2006, 12(10), 1147-1150.

[25] Sampaolesi M.; Torrente Y.; Innocenzi A.; Tonlorenzi R.; D'Antona G.; Pellegrino M.A.; Barresi R.; Bresolin N.; De Angelis M.G.; Campbell K.P.; Bottinelli R.; Cossu G. Cell therapy of alpha-sarcoglycan null dystrophic mice through intra- arterial delivery of mesoangioblasts. Science 2003, 301(5632), 487-492.

[26] Quattrocelli M.; Swinnen M.; Giacomazzi G.; Camps J.; Barthélemy I.; Ceccarelli G.; Caluwé E.; Grosemans H.; Thorrez L.; Pelizzo G.; Muijtjens M.; Verfaillie CM.; Blot S.; Janssens S.; Sampaolesi M. J Clin Invest 2015 125(12), 4463-4482.

[27] Cossu G.; Previtali S.C.; Napolitano S.; Cicalese M.P.; Tedesco F.S.; Nicastro F.; Noviello M.; Roostalu U.; Natali Sora M.G.; Scarlato M.; De Pellegrin M.; Godi C.; Giuliani S.; Ciotti F.; Tonlorenzi R.; Lorenzetti I.; Rivellini C.; Benedetti S.; Gatti R.; Marktel S.; Mazzi B.; Tettamanti A.; Ragazzi M.; Imro M.A.; Marano G.; Ambrosi A.; Fiori R.; Sormani M.P.; Bonini C.; Venturini M.; Politi L.S.; Torrente Y.; Ciceri F. Intra-arterial transplantation of HLA-matched donor mesoangioblasts in Duchenne muscular dystrophy. Embo Mol Med 2015, 7(12), 1513-2. 